\title{
First-principles calculations of dielectric and optical properties of $\mathrm{MgB}_{2}$
}

\author{
A. Balassis, ${ }^{1}$ E. V. Chulkov, ${ }^{1,2}$ P. M. Echenique, ${ }^{1,2}$ and V. M. Silkin ${ }^{1,2}$ \\ ${ }^{1}$ Donostia International Physics Center (DIPC), P. Manuel de Lardizabal, 20018 San Sebastián, Basque Country, Spain \\ ${ }^{2}$ Departamento de Física de Materiales and Centro Mixto CSIC-UPV/EHU, Facultad de Ciencias Químicas, Universidad del País Vasco, \\ Apdo. 1072, 20080 San Sebastián, Basque Country, Spain
}

(Received 21 May 2008; published 2 December 2008)

\begin{abstract}
We report on the results of calculation of low-energy dielectric response and optical properties of $\mathrm{MgB}_{2}$. The calculations have been performed with full inclusion of the $a b$ initio electron band structure making use of random-phase and time-dependent local-density approximations. The role of local-field and exchangecorrelation effects in $\mathrm{MgB}_{2}$ dielectric function is thoroughly examined. Index of refraction, extinction coefficient, and normal-incidence reflectivity exhibiting strong anisotropy were calculated for electromagnetic waves polarized along the $a^{*}$ and $c^{*}$ axes by using the evaluated electron-density-response functions. Our results are in good agreement with recent $\mathrm{x}$-ray and optical measurements performed on $\mathrm{MgB}_{2}$ single crystals.
\end{abstract}

DOI: 10.1103/PhysRevB.78.224502

PACS number(s): 71.45.Gm, 74.70.Ad, 74.25.Gz, 74.25.Jb

\section{INTRODUCTION}

The discovery ${ }^{1}$ of superconductivity in $\mathrm{MgB}_{2}$ at remarkably high transition temperature $T_{C} \sim 39 \mathrm{~K}$ has stimulated numerous theoretical and experimental studies devoted to detailed analysis of its electronic structure, electron-phonon coupling, atomic vibrations, etc. The important part in the investigations of superconducting properties of this compound has been based on the use of $a b$ initio methods and, in particular, on its calculated band structure, ${ }^{2-5}$ which was found to be consistent with photoemission measurements. ${ }^{6-13}$ On the other hand, during the initial period a worse agreement between normal-state optical properties of $\mathrm{MgB}_{2}$ obtained in early spectroscopic works on policrystals ${ }^{14-18}$ and oriented films ${ }^{19,20}$ with the ones obtained on base of $a b$ initio band structure posed some problems. Optical spectroscopy is a powerful tool to measure important parameters such as scattering rate, plasma frequency, and superconducting gap. Thus the plasma frequency $\omega_{p}$ is an important electrodynamic quantity, providing possibility to compare calculated band structure with experiment. Therefore the fact that significantly smaller values of plasma frequencies obtained from optical studies in comparison with those derived from the band-structure calculations $s^{2,3,21}$ was rather puzzling. Finally, recent optical experiments ${ }^{22-25}$ have resolved major problems in this respect, establishing that this discrepancy was mainly due to the strong distortion of the measured spectra at optical frequencies by the presence of electronic collective modes, predicted by ab initio calculations ${ }^{26,27}$ soon after the discovery of superconductivity in $\mathrm{MgB}_{2}$.

During the last years, several experimental and theoretical groups reported on the collective electronic excitations in this material, which exhibits peculiar features related to its band structure. The $\mathrm{MgB}_{2}$ energy band structure presents strong anisotropy related to its atomic lattice. $\mathrm{MgB}_{2}$ crystallizes in a hexagonal-layered structure formed by alternating graphitelike honeycomb boron layers and hexagonal $\mathrm{Mg}$ planes. Its band structure at the Fermi level is characterized by two distinct electronic bands coming almost entirely from the boron states: the almost two-dimensional $\sigma$ bands, formed by hybridized $s-p_{x, y}$ orbitals, and the three- dimensional $\pi$ bands of $p_{z}$ symmetry. ${ }^{2,4,21}$ This strong anisotropy of the band structure in $\mathrm{MgB}_{2}$ is reflected quite vividly in its anisotropic low-energy electronic excitation spectra. Thus a long-lived collective mode between $2.5-4.5 \mathrm{eV}$ for momentum transfer along the $c^{*}$ axis ${ }^{28}$ was predicted in Refs. 26 and 27. It results from interband transitions between boron $\pi$ and magnesium $\zeta$ electron bands ${ }^{27}$ (see Fig. 1) that have almost parallel dispersions in the $\Gamma K M$ plane of the first Brillouin zone (BZ). At small momentum transfer along the $a^{*}$ and $b^{*}$ axes the corresponding mode has significantly smaller energy ${ }^{26}$ due to the $\sigma$ - $\pi$ interband transitions. The signatures of those collective modes were observed in the early electron energy-loss ${ }^{29}$ and photoemission microscopy ${ }^{7}$ experiments. Joint theoretical and experimental study of the plasmon structure in $\mathrm{MgB}_{2}$ at small momenta ${ }^{30}$ detected in $\mathrm{MgB}_{2}$ a peak at $2.4 \mathrm{eV}$ and several peaks for higher energies. A thorough experimental analysis of the energy-loss peak positions in the excitation spectra has been performed in Ref. 31. In general, a good agreement with the $a b$ initio peaks positions ${ }^{26,27}$ was observed, and the strong anisotropy of the $\mathrm{MgB}_{2}$ dielectric response properties was confirmed. It was

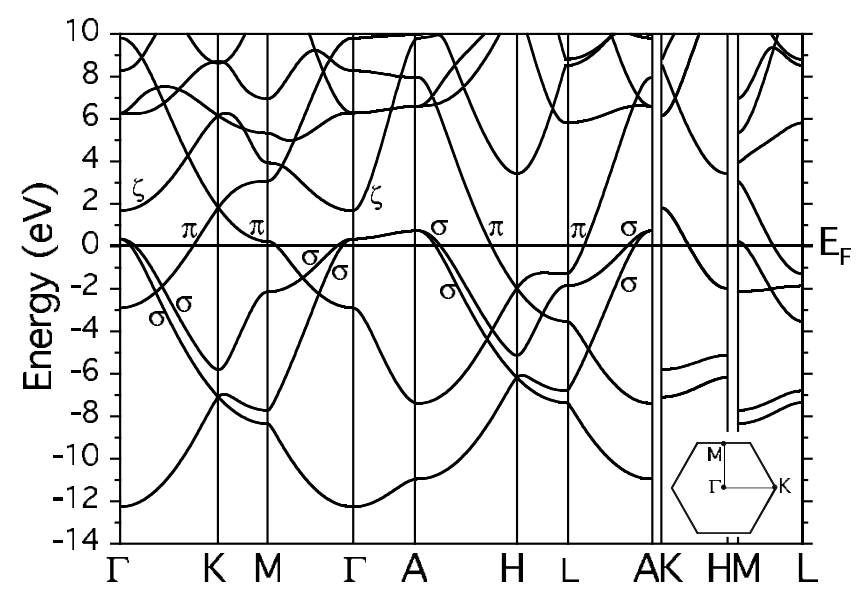

FIG. 1. Band structure of $\mathrm{MgB}_{2}$ along the high-symmetry directions of the first BZ. The Fermi level is at zero energy. The inset shows the symmetry points and directions in the hexagonal plane at $k_{z}=0$. 
concluded $^{31}$ that agreement between theoretically calculated and experimental peak positions is at the same level as the uncertainties in both the calculations and the experiment. Nevertheless, some discrepancies have been pointed out and additional theoretical analysis has been proposed. Very recently joint experimental and theoretical studies $^{32}$ have added further information on the peculiar dielectric properties of $\mathrm{MgB}_{2}$, showing how the long-lived collective mode in the 2.5-4.5 eV energy range evolves with a cosinelike oscillating dispersion at large momenta along the $c^{*}$ axis. Recent optical experiments ${ }^{22,25}$ reported that because of these collective modes the color of $\mathrm{MgB}_{2}$ samples depends on the polarization of the light being bluish silver for $\mathrm{E} \| a^{*}$ polarization and yellow for $\mathrm{E} \| c^{*}$ one. Very recently, in another publication, ${ }^{33}$ on base of the existence of these modes, $\mathrm{MgB}_{2}$ has been proposed as a component of a negative-index metamaterial at visible frequencies with interesting photonic applications.

Despite the extensive theoretical study of the collective electronic modes in $\mathrm{MgB}_{2}$ some relevant issues have not been investigated in detail yet. To the best of our knowledge, an explicit analysis of how local-field and exchangecorrelation effects influence its dielectric and optical properties and, in particular, how they affect the collective charge excitations does not exist. In few publications these topics have only been addressed partially. For instance, Zhukov et $a l .{ }^{26}$ studied collective modes in $\mathrm{MgB}_{2}$ along different momentum directions within the random-phase approximation (RPA) with full inclusion of local-field effects. Later on Cai et al. ${ }^{32}$ demonstrated the decisive role of local-field effects in the peculiar oscillating dispersion of the $2.5-4.5 \mathrm{eV}$ mode at large momenta along the $c^{*}$ axis. On the other hand, from the excellent agreement between the energy of this mode at small momenta obtained within both the RPA (Refs. 26 and 27) and the time-dependent local-density approximation (TDLDA) (Ref. 32) one can figure out that the role of exchange correlations is not important, but the whole picture cannot build up. Additionally, whereas the strong anisotropy of the dielectric properties in magnesium diboride in perpendicular and parallel directions has been established, ${ }^{26,31}$ the detailed momentum-resolved analysis in the parallel plane is still missing.

It is the purpose of the present paper to perform detailed theoretical investigation within an $a b$ initio approach of the excitation spectra in $\mathrm{MgB}_{2}$ for all three symmetry momentum directions. The role of local-field and exchangecorrelation effects beyond the RPA in the dielectric and optical properties of this material is explicitly investigated as well.

The paper is organized as follows: In Sec. II the calculation procedure is described. In Sec. III we present the main results,, which include the $\mathrm{MgB}_{2}$ loss spectra along the main three symmetry momentum directions. The data for the anisotropic optical constants for this compound are presented in this section as well. In Sec. IV we summarize the obtained results. Atomic units $\left(\hbar=e^{2}=m_{e}=1\right)$ are used unless otherwise stated.

\section{CALCULATION DETAILS}

A key quantity in the description of both single-particle and collective electronic excitations in a many-electron sys- tem is the dynamical structure factor $S(\mathbf{q}+\mathbf{G}, \omega),{ }^{34}$ which determines the rate to transfer momentum $\mathbf{q}+\mathbf{G}$ and energy $\omega$ to the periodic solid. Here the wave vector $\mathbf{q}$ is in the first $\mathrm{BZ}$ and $\mathbf{G}$ is a reciprocal lattice vector. In linear-response theory the fluctuation-dissipation theorem relates the dynamical structure factor to the Fourier transform of the density-response function $\chi\left(\mathbf{r}, \mathbf{r}^{\prime}, \omega\right)$ of an interacting electron system as follows:

$$
S(\mathbf{q}+\mathbf{G}, \omega)=-2 \Omega \operatorname{Im}\left[\chi_{\mathbf{G G}}(\mathbf{q}, \omega)\right]
$$

where $\Omega$ is the normalization volume. In general, $\chi\left(\mathbf{r}, \mathbf{r}^{\prime}, \omega\right)$ is a nonlocal energy-dependent function. In the timedependent perturbation theory an integral equation ${ }^{35}$

$$
n^{\text {ind }}(\mathbf{r}, \omega)=\int d \mathbf{r}^{\prime} \chi\left(\mathbf{r}, \mathbf{r}^{\prime}, \omega\right) V^{\text {ext }}\left(\mathbf{r}^{\prime}, \omega\right)
$$

determines the induced electron density $n^{\text {ind }}(\mathbf{r}, \omega)$ in the electron system caused by an external potential $V^{\text {ext }}(\mathbf{r}, \omega)$. Within the framework of time-dependent density-functional theory $^{36,37} \chi\left(\mathbf{r}, \mathbf{r}^{\prime}, \omega\right)$ obeys the integral equation

$$
\begin{aligned}
\chi\left(\mathbf{r}, \mathbf{r}^{\prime}, \omega\right)= & \chi^{0}\left(\mathbf{r}, \mathbf{r}^{\prime}, \omega\right)+\int d \mathbf{r}_{1} \int d \mathbf{r}_{2} \chi^{0}\left(\mathbf{r}, \mathbf{r}_{1}, \omega\right) \\
& \times\left[v\left(\mathbf{r}_{1}-\mathbf{r}_{2}\right)+K^{\mathrm{xc}}\left(\mathbf{r}_{1}, \mathbf{r}_{2}, \omega\right)\right] \chi\left(\mathbf{r}_{2}, \mathbf{r}^{\prime}, \omega\right),
\end{aligned}
$$

where $\chi^{0}\left(\mathbf{r}, \mathbf{r}^{\prime}, \omega\right)$ is the density-response function for the noninteracting electron system, $v\left(\mathbf{r}-\mathbf{r}^{\prime}\right)$ is the bare Coulomb potential, and $K^{\mathrm{xc}}\left(\mathbf{r}, \mathbf{r}^{\prime}, \omega\right)$ accounts for dynamical exchangecorrelation effects. In the present work we perform calculations with the use of two forms of the kernel $K^{\mathrm{xc}}$, namely, RPA, i.e., when $K^{\mathrm{xc}}=0$ and adiabatic extension of TDLDA, ${ }^{38}$ in which $K^{\mathrm{xc}}\left(\mathbf{r}, \mathbf{r}^{\prime}, \omega\right)$ is energy independent and approximated by the functional derivative of the exchangecorrelation potential evaluated within local-density approximation (LDA)

$$
K_{\mathrm{TDLDA}}^{\mathrm{xc}}\left(\mathbf{r}, \mathbf{r}^{\prime}\right)=\delta\left(\mathbf{r}-\mathbf{r}^{\prime}\right) \frac{\partial V_{\mathrm{LDA}}^{\mathrm{xc}}[\rho(\mathbf{r}), \mathbf{r}]}{\partial \rho(\mathbf{r})} .
$$

The LDA exchange-correlation potential, $V_{\mathrm{LDA}}^{\mathrm{xc}}$, is obtained as a result of the $a b$ initio band-structure calculation. In the case of a periodic crystal one can expand all the involved quantities in Fourier series. Hence the integral in Eq. (3) transforms into a matrix equation

$$
\begin{aligned}
\chi_{\mathbf{G G}^{\prime}}(\mathbf{q}, \omega)= & \chi_{\mathbf{G G}^{\prime}}^{0}(\mathbf{q}, \omega)+\sum_{\mathbf{G}_{1}} \sum_{\mathbf{G}_{2}} \chi_{\mathbf{G G}_{1}}^{0}(\mathbf{q}, \omega) \\
& \times\left[v_{\mathbf{G}_{1}}(\mathbf{q}) \delta_{\mathbf{G}_{1} \mathbf{G}_{2}}+K_{\mathbf{G}_{1} \mathbf{G}_{2}}^{\mathrm{xc}}(\mathbf{q}, \omega)\right] \chi_{\mathbf{G}_{2} \mathbf{G}^{\prime}}(\mathbf{q}, \omega) .
\end{aligned}
$$

The Fourier coefficients $\chi_{\mathbf{G G}^{\prime}}^{0}(\mathbf{q}, \omega)$ can be calculated numerically with the use of several methods. ${ }^{39-43}$ Here we use an approach based on the evaluation of a spectral function $S_{\mathbf{G G}^{\prime}}^{0}(\mathbf{q}, \omega)$ as follows: ${ }^{40,44}$ 


$$
\begin{aligned}
S_{\mathbf{G G}^{\prime}}^{0}(\mathbf{q}, \omega)= & \frac{2}{\Omega} \sum_{\mathbf{k}} \sum_{n} \sum_{n^{\prime}}\left\langle\psi_{n \mathbf{k}}\left|e^{-\mathrm{i}(\mathbf{q}+\mathbf{G}) \cdot \mathbf{r}}\right| \psi_{n^{\prime} \mathbf{k}+\mathbf{q}}\right\rangle \\
& \times\left\langle\psi_{n^{\prime} \mathbf{k}+\mathbf{q}}\left|e^{\mathrm{i}\left(\mathbf{q}+\mathbf{G}^{\prime}\right) \cdot \mathbf{r}}\right| \psi_{n \mathbf{k}}\right\rangle \delta\left(\varepsilon_{n \mathbf{k}}-\varepsilon_{n^{\prime} \mathbf{k}+\mathbf{q}}+\omega\right) .
\end{aligned}
$$

Here the factor 2 accounts for the spin, $n$ and $n^{\prime}$ are the band indices, and $\varepsilon_{n \mathbf{k}}$ and $\psi_{n \mathbf{k}}(\mathbf{r})$ are Bloch eigenvalues and eigenfunctions of the Kohn-Sham Hamiltonian. The wave vector $\mathbf{k}$ is confined in the first BZ. In practice, the matrix elements of $S_{\mathbf{G G}^{\prime}}^{0}(\mathbf{q}, \omega)$ are evaluated by replacing the $\delta$ function by a Gaussian with some small broadening. In present work we fix this broadening value to be $0.1 \mathrm{eV}$. Once one has obtained $S_{\mathbf{G G}^{\prime}}^{0}(\mathbf{q}, \omega)$, the matrix elements of the imaginary part of $\chi_{\mathbf{G G}^{\prime}}^{0}(\mathbf{q}, \omega)$ are calculated through the equation

$$
S_{\mathbf{G G}^{\prime}}^{0}(\mathbf{q}, \omega)=\frac{1}{\pi} \operatorname{sgn}(\omega) \operatorname{Im}\left[\chi_{\mathbf{G G}^{\prime}}^{0}(\mathbf{q}, \omega)\right],
$$

while the real part of the $\chi_{\mathbf{G G}^{\prime}}^{0}(\mathbf{q}, \omega)$ matrix elements are obtained from the corresponding imaginary part by the Hilbert transform with the use of the energy cutoff of $50 \mathrm{eV} . \chi^{0}$ is related to the dielectric function, $\epsilon$, as $\epsilon=1-v \chi^{0} /(1$ $\left.-K_{\mathrm{xc}} \chi^{0}\right)$. Finally, the loss function, $-\operatorname{Im}\left[\epsilon_{\mathbf{G G}^{\prime}}^{-1}(\mathbf{q}, \omega)\right]$, is given in terms of the density-response function, $\chi$, by the equation

$$
\epsilon_{\mathbf{G G}^{\prime}}^{-1}(\mathbf{q}, \omega)=\delta_{\mathbf{G G}^{\prime}}+v_{\mathbf{G}}(\mathbf{q}) \chi_{\mathbf{G G}^{\prime}}(\mathbf{q}, \omega) .
$$

The index of refraction, $n$, and the extinction coefficient, $\kappa$, are related to the real and imaginary parts of the macroscopic dielectric function

$$
\epsilon^{M}(\omega)=\lim _{\mathbf{q} \rightarrow 0} \frac{1}{\epsilon_{\mathbf{G}=0 \mathbf{G}^{\prime}=0}^{-1}(\mathbf{q}, \omega)}
$$

through the equations

$$
\begin{gathered}
\operatorname{Re} \epsilon^{M}=n^{2}-\kappa^{2}, \\
\operatorname{Im} \epsilon^{M}=2 n \kappa,
\end{gathered}
$$

and the normal-incidence reflectivity, $R$, is defined by the Fresnel equation

$$
R=\frac{(n-1)^{2}+\kappa^{2}}{(n+1)^{2}+\kappa^{2}} .
$$

Present calculations have been performed for the hexagonal lattice with the $\mathrm{MgB}_{2}$ experimental lattice parameters $a$ $=5.8317$ a.u. and $c=6.6594$ a.u. The one-particle energies $\varepsilon_{n \mathbf{k}}$ and wave functions $\psi_{n \mathbf{k}}(\mathbf{r})$ were evaluated as a selfconsistent solution of the Kohn-Sham equations with the use of exchange-correlation potential $V_{\mathrm{LDA}}^{\mathrm{xc}}$ in the form of Ref. 45. In our calculations the core-valence interaction was taken into account by means of nonlocal norm-conserving pseudopotentials for $\mathrm{Mg}$ and $\mathrm{B}$ generated according to Ref. 46. Valence electronic wave functions, $\psi_{n \mathbf{k}}(\mathbf{r})$, were expanded in a plane-wave basis with a kinetic-energy cutoff of $24 \mathrm{Ry}$. In the Fourier expansion of the $\chi^{0}, \chi$, and $\epsilon$ matrices we included 25 reciprocal vectors $\mathbf{G}$, which ensures convergence of the results obtained. In Eq. (6) the sum over $\mathbf{k}$ includes a $108 \times 108 \times 80$ sampling that corresponds to 933120 points in the first BZ. ${ }^{47}$ The sum over $n$ and $n^{\prime}$ includes all occupied and unoccupied energy bands up to energy of $50 \mathrm{eV}$ above the Fermi level. Note, that in the present calculations the local-field effects are incorporated through the inclusion of nondiagonal matrix elements in the evaluation of $\chi_{\mathbf{G G}^{\prime}}(\mathbf{q}, \omega)$ through Eq. (5).

\section{CALCULATION RESULTS AND DISCUSSION}

\section{A. $\mathrm{MgB}_{2}$ dielectric properties}

The electronic band structure is the starting point in our investigation of the dielectric properties of $\mathrm{MgB}_{2}$. In Fig. 1 we show the evaluated band structure of this compound along various symmetry directions of the BZ, which is in agreement with the previous calculations. ${ }^{2-4,26,27}$ In the figure two boron $p_{x, y}$-derived bands and two boron $p_{z}$-derived bands crossing the Fermi level are marked by $\sigma$ and $\pi$ symbols, respectively. The unoccupied free-electron-like band corresponding to a conducting state of magnesium of $s$ symmetry is depicted in the figure by $\zeta$.

In Figs. 2-4 we present the loss function, $-\operatorname{Im}\left[\epsilon_{\mathbf{G}=0 \mathbf{G}^{\prime}=0}^{-1}(\mathbf{q}, \omega)\right]$, as a function of energy $\omega$ along the three perpendicular directions of momentum transfer $\mathbf{q}$. These data have been obtained with the use of the RPA kernel. Comparison of these three figures shows that the excitation spectra in the $c^{*}$ direction is very distinct from that in the $a^{*} b^{*}$ plane, in agreement with previous $a b$ initio calculations $^{26}$ and inelastic $\mathrm{x}$-ray scattering (IXS) experiment. ${ }^{31}$ Along the $c^{*}$ axis a very sharp plasmon peak $\mathrm{C}_{1}$ with a cosinelike periodic energy dispersion is clearly seen for energies between $\omega_{b}=2.46 \mathrm{eV}$ and $\omega_{u}=4.48 \mathrm{eV}$, in close agreement with dispersion obtained in recent IXS experiment and $a b$ initio calculation. ${ }^{32}$ In Fig. 4 one can see that the experimental dispersion ${ }^{32}$ of this mode almost coincides with the calculated one for all momentum transfers. This long-lived collective mode was predicted at small $q_{\| c^{*}}$ in $\mathrm{MgB}_{2}$ soon after the discovery of superconductivity in this compound. ${ }^{26,27}$ Subsequent experiment, ${ }^{31}$ whose data are shown by dots in Fig. 4, confirmed its existence and pointed out to its long-lived character for all values of transferred momenta studied. At small $q_{\| c^{*}}$ the narrow $\mathrm{C}_{1}$ mode peak in the loss function, as seen in Fig. 5, has its origin in the sharp onset at $\omega=4.5 \mathrm{eV}$ in the imaginary part of dielectric function due to interband transitions between almost parallel electronic $\pi$ and $\zeta$ bands. $^{27}$ The small value of $\operatorname{Im}[\epsilon]$ at $\omega$ $\simeq 2.5 \mathrm{eV}$, where the zero crossing of $\operatorname{Re}[\epsilon]$ takes place, ensures the appearance of a very narrow peak in the loss function. For comparison, in the upper panel of Fig. 5 we also plot $\operatorname{Im}[\epsilon]$ calculated with the use of other $a b$ initio methods. ${ }^{5,15}$ In general, a good agreement in the interband peaks positions obtained by different groups is observed, although some differences can be detected in the amplitudes. A detailed theoretical analysis of the origin of the remarkable cosinelike periodic energy dispersion of the $\mathrm{C}_{1}$ collective mode with momentum transfer seen in Fig. 4 has been performed in recent publication. ${ }^{32}$ The mechanism behind this 


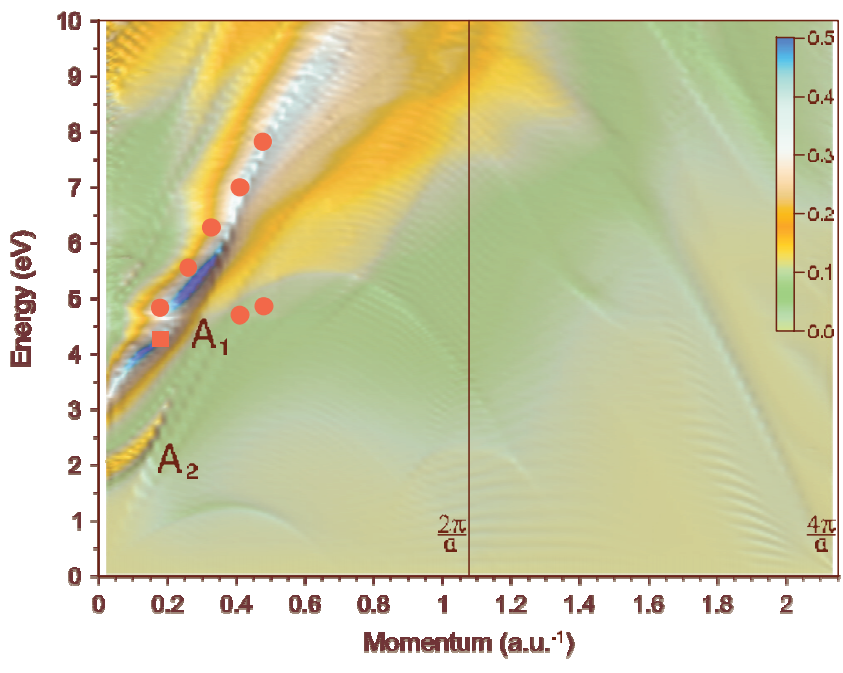

FIG. 2. (Color) Map of $\mathrm{MgB}_{2}$ loss function, $-\operatorname{Im}\left[\epsilon_{\mathbf{G}=0 \mathbf{G}^{\prime}=0}^{-1}(\mathbf{q}, \omega)\right]$, calculated with inclusion of local-field effects within the RPA as a function of energy and momentum transfer $\mathbf{q}_{\| a^{*}}$. Filled circles show the peaks positions in the experimental energyloss spectra from Ref. 31. Filled square marks the peak position extracted from measured spectra reported in Fig. 3(b) of Ref. 31.

behavior is the strong coupling between the collective and single-particle excitation channels mediated by large localfield effects ${ }^{32}$ that redistribute absorption strength from the $\mathrm{C}_{1}$ mode at $q_{\| c^{*}}=Q_{\| c^{*}}$ to the higher $q_{\| c^{*}}=Q_{\| c^{*}}+2 \pi n / c$ harmonics. At the same time, the analysis of Ref. 32 of the physics behind this mode dispersion was restricted to momenta $q_{\| c^{*}}=Q_{\| c^{*}}+2 \pi n / c$ with small value of $Q_{\| c^{*}}$ with $n$ $=0, \pm 1, \ldots$ We note, however, that when $q_{\| c^{*}}$ approaches to $q_{\| c^{*}}=\pi / c$ the situation is remarkably different. In this case, a well-defined plasmon peak does not exist anymore for any

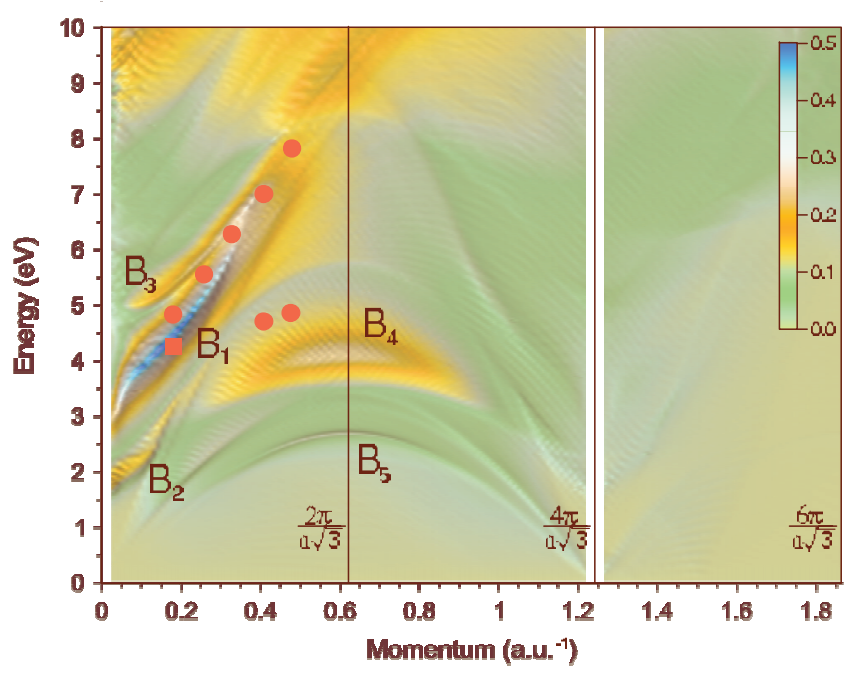

FIG. 3. (Color) Map of $\mathrm{MgB}_{2}$ loss function, $-\operatorname{Im}\left[\epsilon_{\mathbf{G}=0 \mathbf{G}^{\prime}=0}^{-1}(\mathbf{q}, \omega)\right]$, calculated with inclusion of local-field effects within the RPA as a function of energy and momentum transfer $\mathbf{q}_{\| b^{*}}$. Filled circles show the peaks positions in the experimental energyloss spectra from Ref. 31. Filled square marks the peak position extracted from measured spectra reported in Fig. 3(b) of Ref. 31.

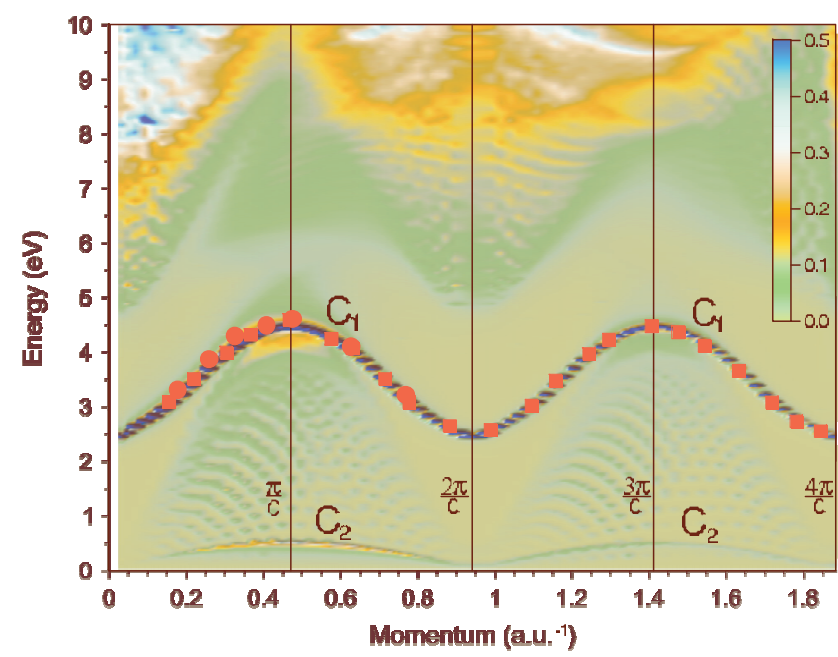

FIG. 4. (Color) Map of $\mathrm{MgB}_{2}$ loss function, $-\operatorname{Im}\left[\epsilon_{\mathbf{G}=0 \mathbf{G}^{\prime}=0}^{-1}(\mathbf{q}, \omega)\right]$, calculated with inclusion of local-field effects within the RPA as a function of energy and momentum transfer $\mathbf{q}_{\| c^{*}}$. Filled circles and squares mark the peaks positions in the experimental energy-loss spectra from Refs. 31 and 32, respectively.

particular matrix element in the dielectric matrix as it is demonstrated in Fig. 6. Indeed, the sharp $\mathrm{C}_{1}$ mode peak disappears in $-\operatorname{Im}\left[\epsilon_{\mathbf{G}=0, \mathbf{G}^{\prime}=0}\left(q_{\| c^{*}}, \omega\right)\right]^{-1}$ (i.e., when local-field effects are not taken into account) at momenta larger than $q_{\| c^{*}} \approx 0.32$ a.u. ${ }^{-1} \cdot{ }^{27}$ In Fig. 6 one can see that the real part of $\epsilon$ does not reach zero in this energy region. This leads to the appearance of only a weak peak in $-\operatorname{Im}\left[\epsilon^{-1}\right]$ around $\omega$ $=3.8 \mathrm{eV}$, i.e., close to $\omega=3.7 \mathrm{eV}$, where the local maximum in $\operatorname{Im}[\epsilon]$ due to the $\pi$ intraband transitions is presented. A dominant peak in the loss function at $\omega=4.48 \mathrm{eV}$ (when the local fields are taken into account) is a result of the presence of a local minimum in $\operatorname{Det}[\epsilon]$ at this energy. The $C_{1}$ peak location in the pseudogap in $\operatorname{Im}[\epsilon]$ between $\omega \sim 4.2 \mathrm{eV}$ and $\omega \sim 5.8 \mathrm{eV}$ ensures its very narrow width, i.e., its long lifetime.

From Fig. 5 one can extract that the $\mathrm{C}_{1}$ mode peak in the loss function is upward shifted by $0.1 \mathrm{eV}$ when the localfield effects are taken into account. This gives evidence that their influence is not only limited by the transmittance of absorption strength from low-momentum harmonics to the higher-momentum ones, but that there also exists some feedback that leads to some increase in the $\mathrm{C}_{1}$ mode frequency. This also leads to some modification of the macroscopic dielectric function (i.e., when the local fields are included) in comparison with the microscopic one (without inclusion of the local fields). This effect is illustrated in the upper panel of Fig. 5, where thick (thin) dashed and solid lines refer to the real and imaginary parts of the dielectric function with (without) taking into account the local-field effects.

A close inspection of Fig. 4 reveals an additional sharp feature in the loss function along the $c^{*}$ axis even for smaller energies. This feature labeled by $\mathrm{C}_{2}$ is clearly seen for all $q_{\| c^{*}}$ and evolves periodically with increasing $q_{\| c^{*}}$ as $\omega_{\mathrm{C}_{2}}$ $=\omega_{\mathrm{C}_{2}}^{\max }\left|\sin \left(\frac{c}{2} q_{\| c^{*}}\right)\right|$. Hence the energy of the $\mathrm{C}_{2}$ mode is proportional to $\left|Q_{\| c^{*}}\right|$ at momenta $q_{\| c^{*}}=Q_{\| c^{*}}+2 \pi n / c$ with small magnitude of $Q_{\| c^{*}}$ and reaches its maximum value of $\omega_{\mathrm{C}_{2}}^{\max }$ 


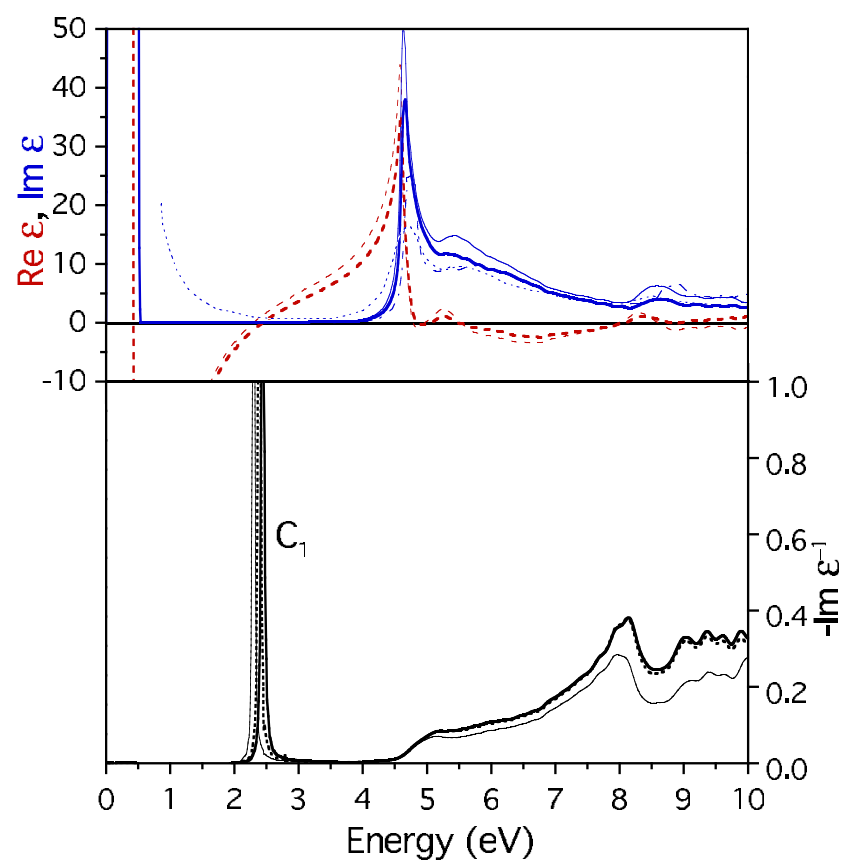

FIG. 5. (Color online) Upper panel: The real (dashed lines) and imaginary (solid lines) parts of the RPA dielectric function calculated at $q_{\|_{c^{*}}}=0.039$ a.u. $^{-1}$. Calculated $\operatorname{Im}[\epsilon(\omega)]$ of Ref. 5 is shown by thin dotted line. The interband contribution to $\operatorname{Im}[\epsilon(\omega)]$ evaluated in Ref. 15 is presented by thin dashed-dotted line. Bottom panel: Corresponding loss function. Solid and dashed lines stand for results of present RPA and TDLDA calculations, respectively. The $\mathrm{C}_{1}$ mode peak is marked by corresponding symbol according to Fig. 4 , while a low-energy peak corresponding the $C_{2}$ mode is not visible on a chosen scale. In both panels thick (thin) solid and dashed lines correspond to the calculations with (without) inclusion of local-field effects.

$=0.5 \mathrm{eV}$ at $q_{\| c^{*}}=\pi / c=0.472$ a.u. $^{-1}$ and $q_{\| c^{*}}=3 \pi / c$ $=1.416$ a.u. $^{-1}$. In Fig. 6 a well-defined peak at $0.35 \mathrm{eV}$ is seen in $\operatorname{Im}[\epsilon]$ at $q_{\| c^{*}}=\pi / c$ due to the intraband transitions in $\sigma$ bands, while $\operatorname{Re}[\epsilon]$ presents only a local minimum at $\omega$ $=0.45 \mathrm{eV}$. Nevertheless, as seen in Fig. 6 this local minimum in $\operatorname{Re}[\epsilon]$ leads to the appearance at almost the same energy of a pronounced peak in the loss function, $-\operatorname{Im}\left[\epsilon^{-1}\right]$, as obtained in the calculation without taking into account the local-field effects. The inclusion of the local-field effects shifts upward this peak to $\omega=0.53 \mathrm{eV}$ and moderately enhances its amplitude. This effect is not as strong as in the case of the upper $\mathrm{C}_{1}$ collective mode, however it is prominent. This low-energy peak has been neither addressed in the previous $a b$ initio studies nor resolved in IXS experiments, ${ }^{31,32}$ where it could be masked by the elastic peak. A more detailed analysis of this feature is given elsewhere. ${ }^{48}$

From comparison of solid and dashed lines in the bottom panels of Figs. 5 and 6, a role played by the exchangecorrelation effects beyond the RPA can be deduced. Figure 5 verifies that at small $q_{\| c}$ *'s their effect on the excitation spectra in $\mathrm{MgB}_{2}$ is small. The only visible effect is a $\sim 0.1 \mathrm{eV}$ downward shift of the peak corresponding to the $\mathrm{C}_{1}$ collective mode, thus bringing its energy in close agreement with the evaluated in Ref. 32. At $q_{\|_{c^{*}}}=0.472$ a.u. $^{-1}$ the situation is

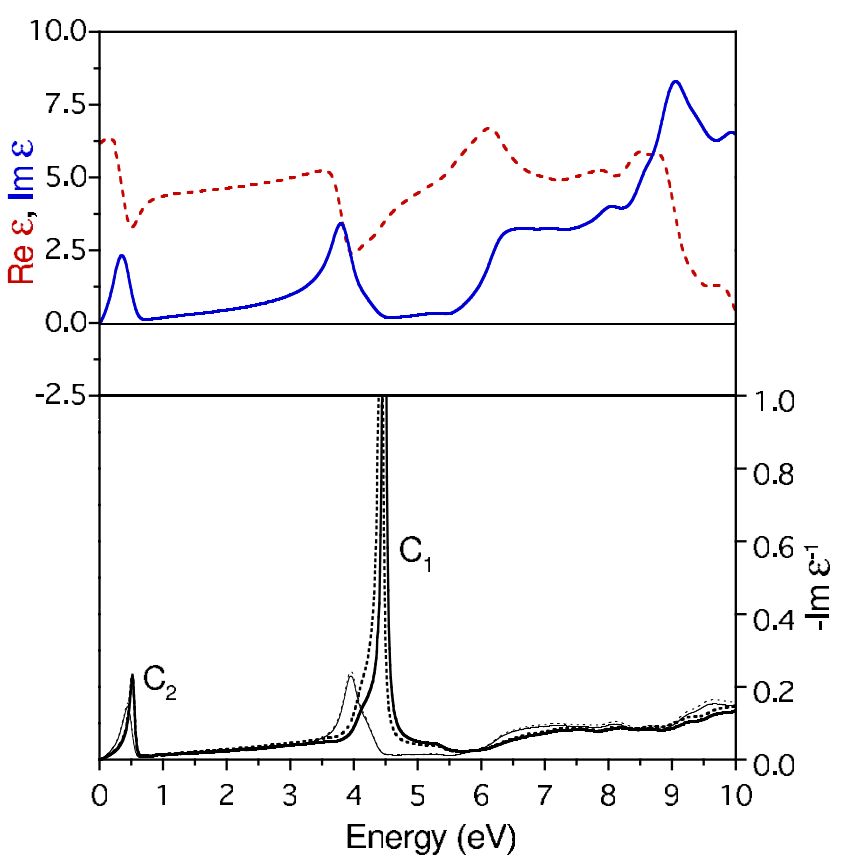

FIG. 6. (Color online) Upper panel: The real (dashed line) and imaginary (solid line) parts of the RPA dielectric function at $q_{\| c^{*}}$ $=0.472$ a.u. ${ }^{-1}$. Bottom panel: Corresponding loss function. Thick (thin) lines correspond to the calculations with (without) inclusion of local-field effects. Solid and dashed lines stand for the results of RPA and TDLDA calculations, respectively. The $\mathrm{C}_{1}$ and $\mathrm{C}_{2}$ mode peaks are marked by corresponding symbols according to Fig. 4.

similar: TDLDA leads to the same $(\sim 0.1 \mathrm{eV})$ downward shift of the $\mathrm{C}_{1}$ mode peak. Additionally, it slightly increases $-\operatorname{Im}\left[\epsilon^{-1}\right]$ for $\omega \leq 4 \mathrm{eV}$ and $\omega \geq 6 \mathrm{eV}$. Hence the use of different exchange-correlation kernels leads only to slight modifications of $-\operatorname{Im}\left[\epsilon^{-1}\right]$, and the main effect of the inclusion of TDLDA kernel is the $\sim 0.1 \mathrm{eV}$ downward shift of the dominant $\mathrm{C}_{1}$ collective-mode peak for all momenta along this symmetry direction.

Comparison of $-\operatorname{Im}\left[\epsilon^{-1}\right]$ along the $a^{*}$ and $b^{*}$ symmetry directions in the hexagonal boron plane reveals a rather surprising and not previously commented fact. In Figs. $2-4$ it is seen that being both very distinct from the excitation spectra along the $c^{*}$ axis, they are also rather distinct from each other. While for small momenta the spectra in Figs. 2 and 3 are very similar, with momentum increase they show different behavior. Along the $a^{*}$ axis the dominant $\mathrm{A}_{1}$ peak disperses from $\omega=3 \mathrm{eV}$ at small momenta (where it is very sharp) up to $\omega \approx 10 \mathrm{eV}$ at $q_{\| a^{*}}=0.8$ a.u. $^{-1}$ acquiring significant width. The dispersion of this peak is in a good agreement with the experimental data ${ }^{31}$ (shown in Fig. 2 by symbols) and the early $a b$ initio calculations. ${ }^{26} \mathrm{~A}$ more detailed inspection of Fig. 2 also reveals that this feature has a pronounced horizontal valley around $\omega=4.6 \mathrm{eV}$ for momenta close to $q=0.2$ a.u. $^{-1}$. As momentum decreases the strength of this mode reduces and another feature labeled by $\mathrm{A}_{2}$ at smaller energy starts to emerge. At $q_{\| a^{*}} \longrightarrow 0$ the latter gains strength very fast and at vanishing $q_{\| a^{*}}$ it is the only dominant peak in the considered energy range. In Fig. 7 the imaginary and real parts of $\epsilon$ and loss function, $-\operatorname{Im}\left[\epsilon^{-1}\right]$, are shown for $q_{\| a^{*}}=0.04$ a.u. $^{-1}$. We also plot in the figure 


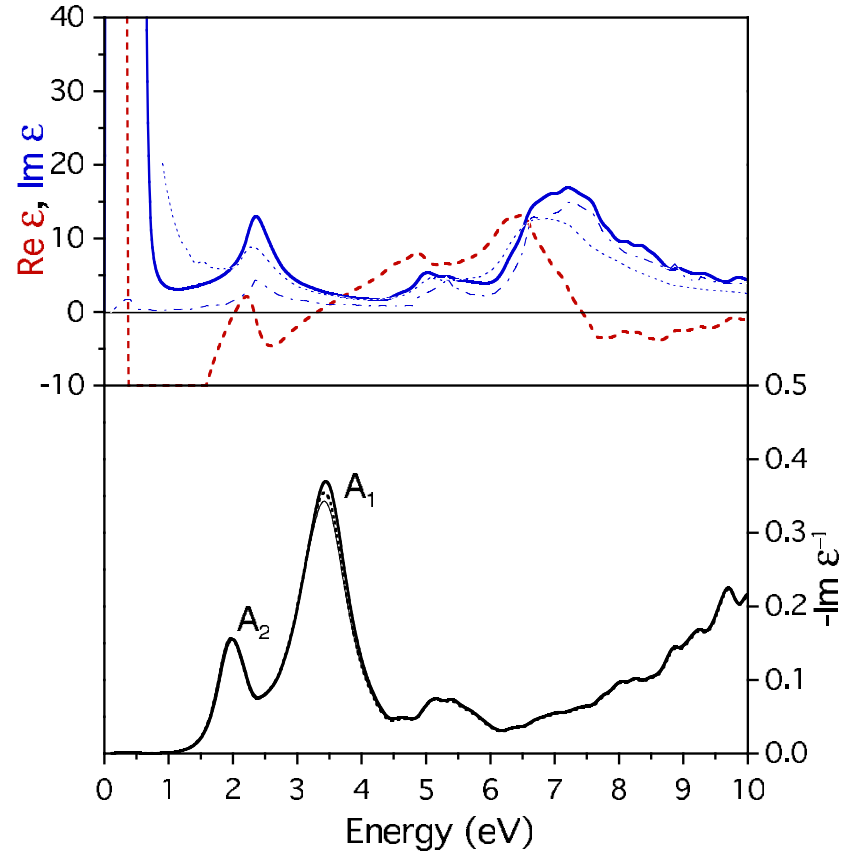

FIG. 7. (Color online) Upper panel: The real (dashed line) and imaginary (solid line) parts of the RPA dielectric function at $q_{\| a^{*}}$ $=0.04$ a.u. ${ }^{-1}$. Calculated $\operatorname{Im}[\epsilon(\omega)]$ of Ref. 5 is shown by thin dotted line. The interband contribution to $\operatorname{Im}[\epsilon(\omega)]$ evaluated in Ref. 15 is presented by thin dashed-dotted line. Bottom panel: Corresponding loss function. Solid and dashed lines stand for results of present RPA and TDLDA calculations, respectively. Thick (thin) lines correspond to the calculations with (without) inclusion of local-field effects. The $A_{1}$ and $A_{2}$ mode peaks are marked by corresponding symbols according to Fig. 2.

$\operatorname{Im}[\epsilon(\omega)]$ calculated in the optical limit by other groups. Again, as in the case of the $c^{*}$ polarization, for the $a^{*}$ polarization different calculations agree very well in reproducing the peaks positions in $\operatorname{Im}[\epsilon]$. The origin of the abovementioned rearrangement of the absorption strength between peaks $A_{1}$ and $A_{2}$ at small momenta is a presence of a peak in $\operatorname{Im}[\epsilon]$ at $\omega=2.4 \mathrm{eV}$ originated from the interband $\sigma-\pi$ transitions in the vicinity of the $M$ point in the BZ. ${ }^{15,49}$ This forces the real part of $\epsilon$ to cross zero three times at $\omega=2$, 2.35 , and $3.3 \mathrm{eV}$, giving rise to the appearance of two peaks in the loss function at $\omega \simeq 2 \mathrm{eV}$ and $\omega \simeq 3.5 \mathrm{eV}$. As seen in Fig. 7 the previous $a b$ initio optical calculations also place the interband $\sigma-\pi$ peak in $\operatorname{Im}[\epsilon]$ at nearly the same energy. ${ }^{5,15,49}$ This $\sigma-\pi$ transition peak was also observed in optical measurements ${ }^{22,24}$ at slightly higher energies. Thus in Ref. 22 this peak was found at $2.6 \mathrm{eV}$ and in Ref. 24 the value of 2.8-2.9 eV has been reported. By passing note, the infrared measurements ${ }^{23}$ reported on the presence of a peak at energies of $0.3-0.5 \mathrm{eV}$ in $\operatorname{Im}[\epsilon]$ in normal state due to the interband transitions between two $\sigma$ bands, which is also present in our calculations.

Figure 2 reveals several additional weak features in the loss function, $-\operatorname{Im}\left[\epsilon^{-1}\right]$, due to intraband and interband transitions. Thus an arc-shaped dispersing peak is centered around $\omega=1 \mathrm{eV}$ at $q_{\| a^{*}} \sim 0.4$ a.u. ${ }^{-1}$. Another notable feature has the negative dispersion at large $q_{\| a^{*}}$ 's and it tends to zero as $q_{\| a^{*}} \rightarrow \frac{4 \pi}{a}=2.155$ a.u. $^{-1}$. An additional broad peak struc-

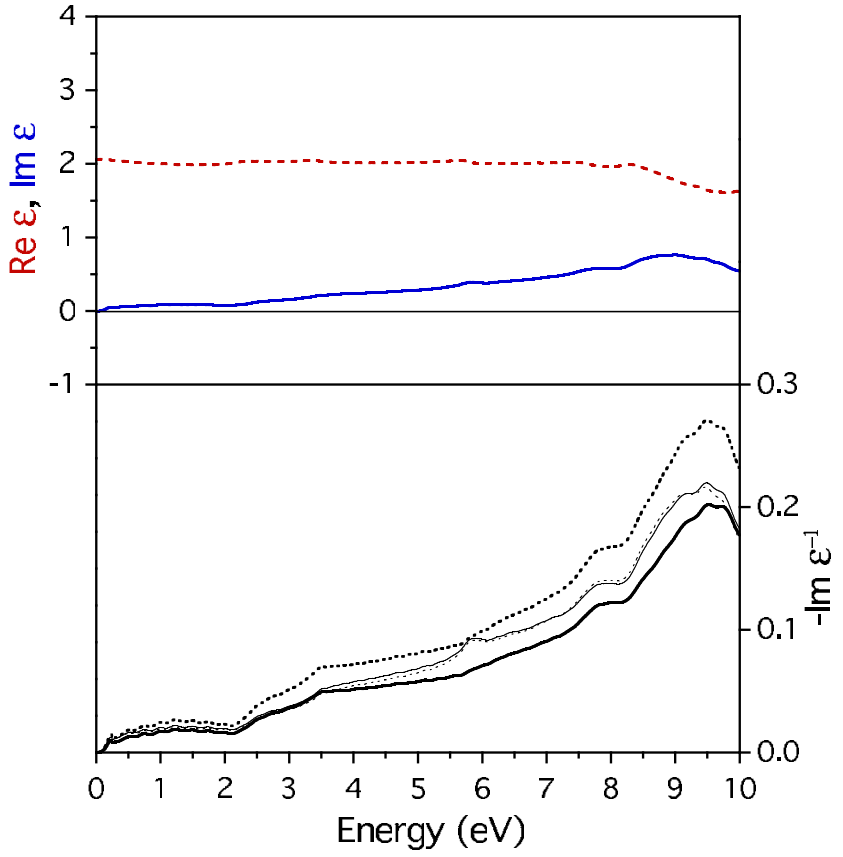

FIG. 8. (Color online) Upper panel: The real (dashed line) and imaginary (solid line) parts of the RPA dielectric function at $q_{\| a^{*}}$ $=1.077$ a.u. ${ }^{-1}$. Bottom panel: Corresponding loss function. Thick (thin) lines correspond to the calculations with (without) inclusion of local-field effects. Solid and dashed lines stand for the results of RPA and TDLDA calculations, respectively.

ture disperses from $\omega \sim 5 \mathrm{eV}$ at $q_{\| a^{*}} \sim 0.4$ a.u. $^{-1}$ up to $\omega$ $\sim 10 \mathrm{eV}$ at $q_{\| a^{*}} \sim 1.1$ a.u. $^{-1}$.

The role of local-field and exchange-correlation effects in the loss function, $-\operatorname{Im}\left[\epsilon^{-1}\right]$, for momentum transfer along the $a^{*}$ axis is demonstrated in Figs. 7 and 8. The bottom panel of Fig. 7 demonstrates that at small momenta both factors for this polarization play only minor role in the formation of the excitation spectra since all four curves are almost nondistinguished on the scale of the figure. The only visible effect is seen around the $\mathrm{A}_{1}$ peak. Figure 8 shows that at large $q_{\| a^{*}}$ 's both those factors lead to moderate changes in the amplitude of $-\operatorname{Im}\left[\epsilon^{-1}\right]$ without significant modification of its shape.

As it was pointed out above, along the $b^{*}$ direction for medium and large momenta $-\operatorname{Im}\left[\epsilon^{-1}\right]$ is notably distinct from that along the $a^{*}$ one. First of all, the dominant $\mathrm{B}_{1}$ peak (with almost the same dispersion al small momenta as $A_{1}$ along the $a^{*}$ axis) disperses along this momentum direction up to $\omega \approx 8 \mathrm{eV}$ and at higher energies it is presented as a quite weak feature. Similar to the $a^{*}$ axis case, at small $q_{\| b^{*}}$ the $\mathrm{B}_{2}$ peak arises at $\omega \approx 2 \mathrm{eV}$. It also disperses upward with increasing momentum and is clearly visible up to $\omega$ $\approx 3.4 \mathrm{eV}$ at $q_{\| b^{*}}=0.2$ a.u. $^{-1}$. An additional weak peak structure (labeled by $\mathrm{B}_{3}$ in Fig. 3) with positive dispersion arises around $\omega \approx 5 \mathrm{eV}$ at $q_{\| b^{*}}=0.05$ a.u. $^{-1}$ and merges to the main $\mathrm{B}_{1}$ peak at $\omega \approx 6 \mathrm{eV}$ around $q_{\| b^{*}}=0.35 \mathrm{a} \mathrm{u}^{-1}$. Also a strong $\mathrm{B}_{4}$ peak, which does not exist along the $a^{*}$ axis, is centered at $\omega=4 \mathrm{eV}$ around $q_{\| b^{*}}=0.6$ a.u. $^{-1}$. Some other weaker peak structures can also be detected in Fig. 3 due to numerous intraband and interband transitions.

In Fig. 9 we present the imaginary and real parts of $\epsilon$ and loss function, $-\operatorname{Im}\left[\epsilon^{-1}\right]$, for $q_{\| b^{*}}=\frac{2 \pi}{a \sqrt{3}}=0.622$ a.u. ${ }^{-1}$, i.e., at 


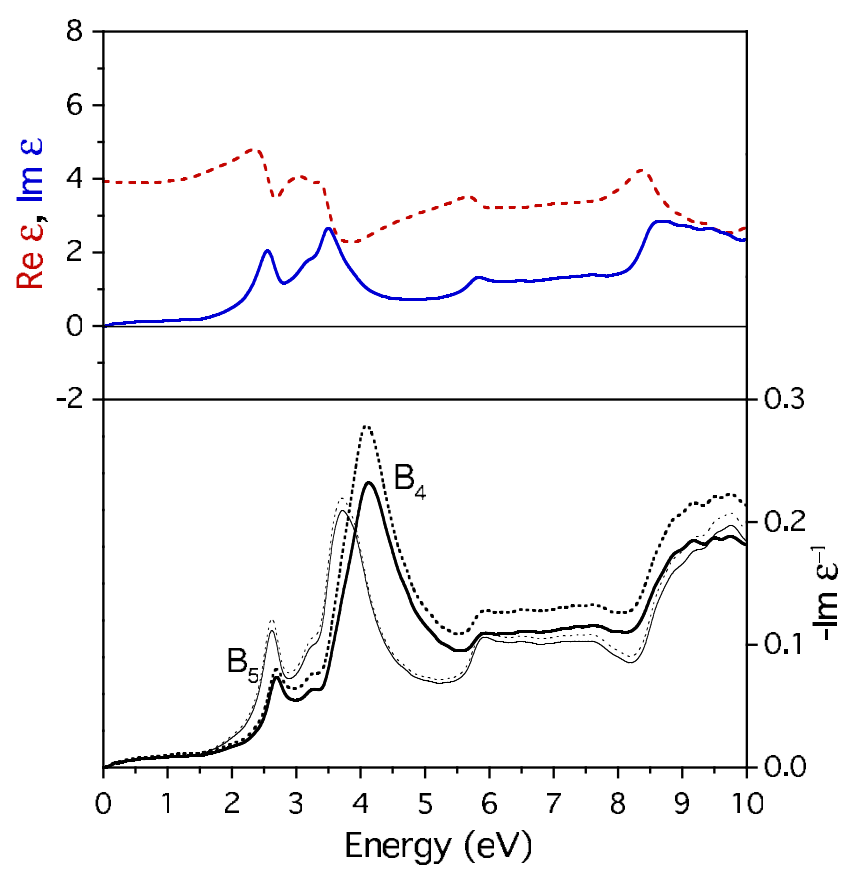

FIG. 9. (Color online) Upper panel: The real (dashed line) and imaginary (solid line) parts of the RPA dielectric function at $q_{\| b^{*}}$ $=0.622$ a.u. ${ }^{-1}$. Bottom panel: Corresponding loss function. Thick (thin) lines correspond to the calculations with (without) inclusion of local-field effects. Solid and dashed lines stand for the results of RPA and TDLDA calculations, respectively. The $\mathrm{B}_{4}$ and $\mathrm{B}_{5}$ mode peaks are marked by corresponding symbols according to Fig. 3.

the border of the first BZ. Again, as in the case of the dielectric function at $q_{\| c^{*}}=\pi / c$, we do not find zero crossing in the real part of $\epsilon$. On the other hand, inclusion of the exchangecorrelation effects within the TDLDA leads to significant enhancement of the peak amplitude of $-\operatorname{Im}\left[\epsilon^{-1}\right]$ around $\omega$ $\approx 4 \mathrm{eV}$ with small downward shift of its energy position. Additionally, the inclusion of the TDLDA kernel at this momentum leads to some increase in $-\operatorname{Im}\left[\epsilon^{-1}\right]$ at all energies. The peak at $\approx 2.7 \mathrm{eV}$ in Fig. 9 is clearly visible in Fig. 3 (the $\mathrm{B}_{5}$ peak structure) as a feature dispersing downward from $q=0.622$ a.u. $^{-1}$ for both decreasing and increasing momenta. Similar to Fig. 2, the dominant $\mathrm{B}_{1}$ peak dispersion along the $b^{*}$ axis is in good agreement with previous calculations ${ }^{26}$ and experimental data. ${ }^{31}$ At the same time, the experimental

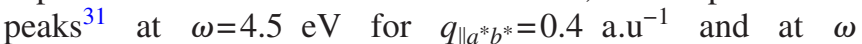
$=4.8 \mathrm{eV}$ for $q_{\| a^{*} b^{*}}=0.45 \mathrm{a} \cdot \mathrm{u}^{-1}$ do not correspond to any theoretically derived peaks in both $a^{*}$ and $b^{*}$ directions. The theoretical peak $\mathrm{B}_{4}$ at $q \sim 0.6$ a.u. ${ }^{-1}$ along the $b^{*}$ axis has not been observed in Ref 31 . We attribute this to the fact that the experiment in Ref. 31 was not angle resolved in the $a^{*} b^{*}$ hexagonal plane.

\section{B. $\mathrm{MgB}_{2}$ optical properties}

We have calculated the optical constants for $\mathrm{MgB}_{2}$ for polarization along all the three symmetry directions through Eqs. (10)-(12) with the use of macroscopic $\epsilon^{M}(q, \omega)$ calculated for small momenta $q$. In Fig. 10 we show the optical data for the $c^{*}$-axis polarization. The very sharp reflectivity

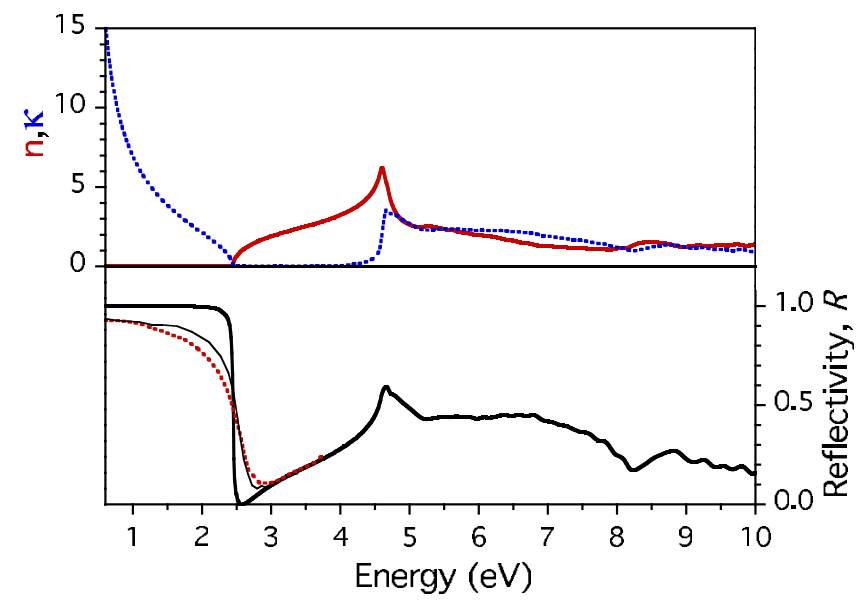

FIG. 10. (Color online) Upper panel: Calculated index of refraction $n(\omega)$ and extinction coefficient $\kappa(\omega)$ for the light polarization along the $c^{*}$-axis direction are shown by solid and dashed lines, respectively. Bottom panel: Calculated for the same polarization normal-incidence reflectivity $R(\omega)$ is shown by thick solid line. The measured reflectivity is presented by thin solid line (Ref. 24) and thick dotted line (Ref. 22). The theoretical curves are evaluated with the use of macroscopic dielectric function $\epsilon^{M}$ for $q_{\| c^{*}}$ $=0.039$ a.u. ${ }^{-1}$.

plasma edge is clearly seen in our data at $2.4 \mathrm{eV}$, in close agreement with other calculations. ${ }^{15,27,49}$ The measured reflectivity plasma edge $\mathrm{e}^{22,24}$ is in fairly good agreement with the calculations for this polarization. Nevertheless the experimental edge is more diffuse. At present it is not clear whether this is the consequence of some imperfections of the $\mathrm{MgB}_{2}$ samples used or due to some intrinsic mechanisms not included in the theory. The calculated dip at $2.7 \mathrm{eV}$ in the reflectivity is followed by a broad region with $R$ reaching 0.4 at $\omega \sim 6 \mathrm{eV}$. At $\omega=4.6 \mathrm{eV}$ the normal reflectivity has a local maximum where it reaches the 0.6 value due to the presence of a peak in $n$ and a sharp onset in $\kappa$ at almost the same frequency. This high-energy behavior of $R$ correlates with that obtained in other calculations, ${ }^{15,27,49}$ although one can note some differences in fine details among data obtained by different groups.

Figure 11 shows refraction index $n$, extinction coefficient $\kappa$, and reflectance $R$ for the light polarization along the $a^{*}$ axis that are also in agreement with other theoretical data. ${ }^{15,49}$ We obtain the same results for the polarization along the $b^{*}$ axis. In our calculation a reflectivity plasma edge for this polarization is at $\sim 1.7 \mathrm{eV}$. With increasing energy the reflectivity reaches the local minimum at $\omega$ $=2.1 \mathrm{eV}$ and its local maximum is observed at $\omega=2.6 \mathrm{eV}$. The calculated reflectivity behavior at the plasma edge is in good agreement with the data of recent optical experiments performed on $\mathrm{MgB}_{2}$ single crystals. ${ }^{22-24}$ Thus the local minimum in the experimentally measured $R$ is observed at $2.3 \mathrm{eV}$, i.e., only at slightly higher energy than our calculated value. Also the positions of the local maximum in the experimental $R$ are close to the calculated one although they are somewhat blueshifted in comparison with our data. In the experiment of Ref. 24 this maximum is centered at $\omega \sim 3 \mathrm{eV}$, and the measurements of Ref. 22 performed for different samples place it 


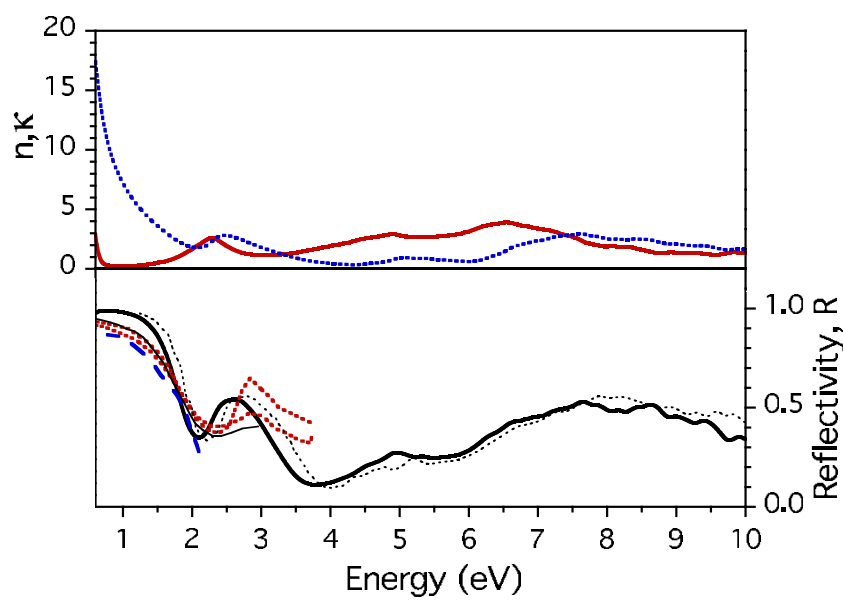

FIG. 11. (Color online) Upper panel: Calculated index of refraction $n(\omega)$ and extinction coefficient $\kappa(\omega)$ for the light polarization along the $a^{*}$ axis are shown by solid and dashed lines, respectively. Bottom panel: Calculated for the same polarization normalincidence reflectivity $R(\omega)$ is shown by thick solid line. The measured reflectivity is presented by thin solid line (Ref. 24), two thick dotted lines (obtained for two different samples) (Ref. 22), and thick dashed line (Ref. 23). The theoretical curves are evaluated with the use of macroscopic dielectric function $\epsilon^{M}$ for $q_{\| a^{*}}$ $=0.04$ a.u. ${ }^{-1}$. Thin dotted line shows $R$ calculated with the use of $\epsilon^{M}$ for $q_{\| a^{*}}=0.08$ a.u. ${ }^{-1}$.

at $\sim 2.8 \mathrm{eV}$ and reveal strong influence of the sample quality on the behavior of $R$ at $\omega \geq 2 \mathrm{eV}$.

The downward shift of the in-plane reflectivity plasma edge by about $0.5 \mathrm{eV}$ with respect to the one along the $c^{*}$ axis is a result of strong $\sigma-\pi$ interband transitions producing peak at $2 \mathrm{eV}$ in $\operatorname{Im}[\epsilon(q \rightarrow 0, \omega)]$ for this polarization as seen in Fig. 7. A slightly redshifted calculation value for these interband transitions with respect to the experiment can be explained by a smaller energy separation between the calculated $\sigma$ and $\pi$ bands at the $M$ point of the BZ. On the other hand, Fig. 11 reveals that the same level of mismatch exists between experimental data obtained on the best quality samples, ${ }^{22-24}$ suggesting that there is a room for further modifications in the experimental data. Detailed inspection of the calculated dielectric functions at small finite momenta in the $a^{*} b^{*}$ plane reveals strong dependence of the evaluated reflectivity $R$ on the momentum value. This is linked to the strong dispersion of the peaks in $-\operatorname{Im}\left[\epsilon^{-1}(q, \omega)\right]$ at small momenta in the $2-4 \mathrm{eV}$ energy range in Figs. 2 and 3. To illustrate this, in the bottom panel of Fig. 11 a thin dashed line shows $R$ evaluated with the use of $\epsilon^{M}(q, \omega)$ for $q$ $=0.08$ a.u. $^{-1}$. One can see how the local maximum in the calculated $R$ shifts from $2.6 \mathrm{eV}$ obtained with the use of $\epsilon^{M}(q, \omega)$ for $q=0.04$ a.u. $^{-1}$ to $2.8 \mathrm{eV}$ in this case. This strong dependence of evaluated $R$ on the used momentum value suggests that the quality of a sample can dramatically affect the position of this maximum in reflectivity and that for even better quality $\mathrm{MgB}_{2}$ samples the blue color observed for this polarization nowadays might acquire some greenish tint.

\section{CONCLUSIONS}

We presented the results of an $a b$ initio study of dielectric and optical properties of $\mathrm{MgB}_{2}$ in the low-energy domain.
The excitation spectra have been investigated for the three symmetry momentum directions. Our calculations reveal that, additionally to a known strong anisotropy in dielectric properties of this compound along the $c^{*}$ axis and in the $a^{*} b^{*}$ plane, remarkable difference also exists between the results for the $a^{*}$ and $b^{*}$ axes. Our analysis showed that the origin of a long-lived $2.5-4.5 \mathrm{eV}$ collective mode along the $c^{*}$ axis depends on the momentum. Whereas transmittance of this mode to the momenta $q_{\| c^{*}}=Q_{\| c^{*}}+2 \pi n / c$ (with $n \neq 0$ ) with small magnitude of $Q_{\| c^{*}}$ is due to large local-field effects, ${ }^{32}$ at $q_{\| c^{*}}=Q_{\| c^{*}}+2 \pi / c$ with $Q_{\| c^{*}} \simeq \pi / c$ the effect of the local fields is even more peculiar: a sharp peak in the loss function is entirely due to these effects as no dielectric matrix element alone has any pole. In the $0-0.5 \mathrm{eV}$ energy domain we found additionally a sharply peaked collective mode, which has a sinelike oscillating dispersion at all momenta. The dispersion of this mode closely follows the upper edge of the region of intraband $\sigma$ transitions and is linear at momenta $q_{\| c^{*}}=Q_{\| c^{*}}$ $+2 \pi n / c$ given $Q_{\|_{c}^{*}}$ are small. In the case of the $0-0.5 \mathrm{eV}$ mode the effect of inclusion of local fields beyond the RPA is also important, making this mode peak more pronounced.

We reported on the substantial anisotropy between loss spectra along the $a^{*}$ and $b^{*}$ axes for finite momenta. At small momenta the position of a dominant mode in these directions is determined by the interband $\sigma-\pi$ transitions in vicinity of the BZ $M$ point. The dominant mode has fairly similar dispersions in both directions, although substantial anisotropy in the dispersion and especially in the width of this mode was observed. The band-structure anisotropy in the $a^{*} b^{*}$ plane reflects itself also in the excitation spectra, leading to other various peaks presented for finite momenta along both $a^{*}$ and $b^{*}$ symmetry directions.

The effect of exchange correlation beyond the RPA has been investigated. Along the $c^{*}$ axis their impact is limited by the downward rigid shift by $0.1 \mathrm{eV}$ of the $2.5-4.5 \mathrm{eV}$ mode at all momenta and small enhancement of $-\operatorname{Im}\left[\epsilon^{-1}\right]$ for the higher energies. In the $a^{*}$ and $b^{*}$ directions the inclusion of exchange correlations within the TDLDA approach produces more notable effects on the loss function, significantly enhancing its amplitude for all energies without appreciable changes in the peaks positions.

In general, the dispersion of the main peaks is in good agreement with the data of the recent IXS experiment, ${ }^{31}$ although some inconsistencies between our calculations and the experiment have been observed, mainly being in the $a^{*} b^{*}$ plane. In order to clarify this it is desirable to perform angleresolved experimental study within this plane too.

The presence of a collective mode in the region of optical frequencies strongly affects the optical properties of $\mathrm{MgB}_{2}$. Our calculated optical constants reveal strong anisotropy between $E \| c^{*}$ and $E \| a^{*} b^{*}$ polarizations. The sharp plasma edge in normal reflectivity for the light with $E \| c^{*}$ polarization is placed at $2.4 \mathrm{eV}$, followed by a region of zero reflectivity at $2.6 \mathrm{eV}$. In the $a^{*} b^{*}$ plane the plasma edge is more diffused and shifted to $\omega \approx 1.7 \mathrm{eV}$. Our optical results are in close agreement with other $a b$ initio calculations and recent experimental optical data obtained with the use of singlecrystal surfaces. 


\section{ACKNOWLEDGMENTS}

We acknowledge the partial support from the University of the Basque Country (Grant No. GIC07IT36607), the De- partamento de Educación del Gobierno Vasco, and the Spanish Ministerio de Ciencia y Tecnología (MCyT) (Grant No. FIS200766711C0101). The work of V.M.S. is sponsored by the IKERBASQUE Foundation.
${ }^{1}$ J. Nagamatsu, N. Nakagawa, T. Muranaka, Y. Zenitani, and J. Akimitsu, Nature (London) 410, 63 (2001).

${ }^{2}$ J. Kortus, I. I. Mazin, K. D. Belashchenko, V. P. Antropov, and L. L. Boyer, Phys. Rev. Lett. 86, 4656 (2001).

${ }^{3}$ Y. Kong, O. V. Dolgov, O. Jepsen, and O. K. Andersen, Phys. Rev. B 64, 020501(R) (2001).

${ }^{4}$ J. M. An and W. E. Pickett, Phys. Rev. Lett. 86, 4366 (2001).

${ }^{5}$ P. Ravindran, P. Vajeeston, R. Vidya, A. Kjekshus, and H. Fjellvåg, Phys. Rev. B 64, 224509 (2001).

${ }^{6}$ H. Uchiyama, K. M. Shen, S. Lee, A. Damascelli, D. H. Lu, D. L. Feng, Z.-X. Shen, and S. Tajima, Phys. Rev. Lett. 88, 157002 (2002).

${ }^{7}$ A. Goldoni, R. Larciprete, S. Lizzit, S. La Rosa, A. Bianco, and M. Bertolo, Phys. Rev. B 66, 132503 (2002).

${ }^{8}$ S. Tsuda, T. Yokoya, Y. Takano, H. Kito, A. Matsushita, F. Yin, J. Itoh, H. Harima, and S. Shin, Phys. Rev. Lett. 91, 127001 (2003).

${ }^{9}$ S. Souma, Y. Machida, T. Sato, T. Takahashi, H. Matsui, S.-C. Wang, H. Ding, A. Kaminski, J. C. Campuzano, S. Sasaki, and K. Kadowaki, Nature (London) 423, 65 (2003).

${ }^{10}$ H. Uchiyama, S. Tajima, K. M. Shen, D. H. Lu, and Z.-X. Shen, Physica C 385, 85 (2003).

${ }^{11}$ G. P. Zhang, G. S. Chang, T. A. Callcott, D. L. Ederer, W. N. Kang, E.-M. Choi, H.-J. Kim, and S.-I. Lee, Phys. Rev. B 67, 174519 (2003).

${ }^{12}$ C. Jariwala, A. Chainani, S. Tsuda, T. Yokoya, S. Shin, Y. Takano, K. Togano, S. Otani, and H. Kito, Phys. Rev. B 68, 174506 (2003).

${ }^{13}$ L. Petaccia, C. Cepek, S. Lizzit, R. Larciprete, R. Macovez, M. Sancrotti, and A. Goldoni, New J. Phys. 8, 12 (2006).

${ }^{14}$ B. Gorshunov, C. A. Kuntscher, P. Haas, M. Dressel, F. P. Mena, A. B. Kuz'menko, D. van der Marel, T. Muranaka, and J. Akimitsu, Eur. Phys. J. B 21, 159 (2001).

${ }^{15}$ A. B. Kuzmenko, F. P. Mena, H. J. A. Molegraaf, D. van der Marel, B. Gorshunov, M. Dressel, I. I. Mazin, J. Kortus, O. V. Dolgov, T. Muranaka, and J. Akimitsu, Solid State Commun. 121, 479 (2002).

${ }^{16}$ Y. Fudamoto and S. Lee, Phys. Rev. B 68, 184514 (2003).

${ }^{17}$ J. H. Jung, S. A. Seo, T. W. Noh, P. A. Sharma, N. Hur, and S.-W. Cheong, Solid State Commun. 126, 175 (2003).

${ }^{18}$ D. Chvostová, V. Železný, L. Pajasová, A. Tarasenko, A. Pleceník, P. Kúš, and L. Satrapinský, Thin Solid Films 455, 213 (2004).

${ }^{19}$ J. J. Tu, G. L. Carr, V. Perebeinos, C. C. Homes, M. Strongin, P. B. Allen, W. N. Kang, E.-M. Choi, H.-J. Kim, and S.-I. Lee, Phys. Rev. Lett. 87, 277001 (2001).

${ }^{20}$ R. A. Kaindl, M. A. Carnahan, J. Orenstein, D. S. Chemla, H. M. Christen, H. Y. Zhai, M. Paranthaman, and D. H. Lowndes, Phys. Rev. Lett. 88, 027003 (2001).

${ }^{21}$ A. Y. Liu, I. I. Mazin, and J. Kortus, Phys. Rev. Lett. 87, 087005
(2001).

${ }^{22}$ V. Guritanu, A. B. Kuzmenko, D. van der Marel, S. M. Kazakov, N. D. Zhigadlo, and J. Karpinski, Phys. Rev. B 73, 104509 (2006).

${ }^{23}$ D. Di Castro, M. Ortolani, E. Cappelluti, U. Schade, N. D. Zhigadlo, and J. Karpinski, Phys. Rev. B 73, 174509 (2006).

${ }^{24}$ T. Kakeshita, S. Lee, and S. Tajima, Phys. Rev. Lett. 97, 037002 (2006).

${ }^{25}$ A. B. Kuzmenko, Physica C 456, 63 (2007).

${ }^{26}$ V. P. Zhukov, V. M. Silkin, E. V. Chulkov, and P. M. Echenique, Phys. Rev. B 64, 180507(R) (2001).

${ }^{27}$ W. Ku, W. E. Pickett, R. T. Scalettar, and A. G. Eguiluz, Phys. Rev. Lett. 88, 057001 (2002).

${ }^{28}$ The $a^{*}, b^{*}$, and $c^{*}$ axes are defined to be parallel to the $\Gamma K, \Gamma M$, and $\Gamma A$ directions of the hexagonal Brillouin zone, respectively (see inset in Fig. 1 for notations in the hexagonal $k_{z}=0$ plane).

${ }^{29}$ V. J. Keast, Appl. Phys. Lett. 79, 3491 (2001).

${ }^{30}$ V. J. Keast, J. Electron Spectrosc. Relat. Phenom. 143, 99 (2005).

${ }^{31}$ S. Galambosi, J. A. Soininen, A. Mattila, S. Huotari, S. Manninen, G. Vankó, N. D. Zhigadlo, J. Karpinski, and K. Hämäläinen, Phys. Rev. B 71, 060504(R) (2005).

${ }^{32}$ Y. Q. Cai, P. C. Chow, O. D. Restrepo, Y. Takano, K. Togano, H. Kito, H. Ishii, C. C. Chen, K. S. Liang, C. T. Chen, S. Tsuda, S. Shin, C. C. Kao, W. Ku, and A. G. Eguiluz, Phys. Rev. Lett. 97, 176402 (2006).

${ }^{33}$ A.-G. Kussow, A. Akyurtlu, A. Semichaevsky, and N. Angkawisittpan, Phys. Rev. B 76, 195123 (2007).

${ }^{34}$ D. Pines and P. Nozières, The Theory of Quantum Liquids (Benjamin, New York, 1966).

${ }^{35}$ A. L. Fetter and J. D. Walecka, Quantum Theory of ManyParticle Systems (McGraw-Hill, New York, 1971).

${ }^{36}$ E. Runge and E. K. U. Gross, Phys. Rev. Lett. 52, 997 (1984).

${ }^{37}$ M. Petersilka, U. J. Gossmann, and E. K. U. Gross, Phys. Rev. Lett. 76, 1212 (1996).

${ }^{38}$ E. K. U. Gross, F. J. Dobson, and M. Petersilka, Density Functional Theory (Springer, Berlin, 1996).

${ }^{39}$ N. E. Maddocks, R. W. Godby, and R. J. Needs, Europhys. Lett. 27, 681 (1994).

${ }^{40}$ F. Aryasetiawan and O. Gunnarsson, Phys. Rev. B 49, 16214 (1994).

${ }^{41}$ W. Ku and A. G. Eguiluz, Phys. Rev. Lett. 82, 2350 (1999).

${ }^{42}$ F. Aryasetiawan, in Strong Coulomb Correlations in Electronic Structure Calculations, edited by V. I. Anisimov (Gordon and Breach, Singapore, 2001).

${ }^{43}$ J. M. Pitarke, V. M. Silkin, E. V. Chulkov, and P. M. Echenique, Rep. Prog. Phys. 70, 1 (2007).

${ }^{44}$ V. M. Silkin, I. P. Chernov, P. M. Echenique, Yu. M. Koroteev, and E. V. Chulkov, Phys. Rev. B 76, 245105 (2007).

${ }^{45}$ D. M. Ceperley and B. J. Alder, Phys. Rev. Lett. 45, 566 (1980); 
as parametrized by J. P. Perdew and A. Zunger, Phys. Rev. B 23, 5048 (1981).

${ }^{46}$ N. Troullier and J. L. Martins, Phys. Rev. B 43, 1993 (1991).

${ }^{47}$ In the optical constants evaluation we employ in sum over $\mathbf{k}$ of Eq. (6) the two to three times more dense meshes.
${ }^{48}$ V. M. Silkin, A. Balassis, P. M. Echenique, and E. V. Chulkov, arXiv:0805.1558 (unpublished).

${ }^{49}$ V. P. Antropov, K. D. Belashchenko, M. van Schilfgaarde, and S. N. Rashkeev, Studies of High Temperature Superconductors, edited by A. Narlikar (Nova Science, New York, 2001). 